International Journal of Computer Networks \& Communications (IJCNC) Vol.3, No.6, November 2011

\title{
Range Free Localization Schemes for Wireless Sensor Networks
}

\author{
Ashok Kumar ${ }^{1}$, Narottam Chand ${ }^{2}$, Vinod Kumar ${ }^{1}$ and Vinay Kumar ${ }^{1}$ \\ ${ }^{1}$ Department of Electronics and Communication Engineering \\ ${ }^{2}$ Department of Computer Science and Engineering \\ National Institute of Technology \\ Hamirpur, INDIA \\ Email: ashok.nithamegmail.com
}

\begin{abstract}
Wireless sensor networks have become a hot research area due to their numerous applications in monitoring inaccessible areas, which are difficult to be monitored by conventional methods. A large number of wireless sensor network based applications are location dependent, where the sensed data is meaningless without accurate location of its origin. Some of these applications require highly accurate location information of sensor nodes. However, in many applications, knowledge of coarse accuracy of sensor node localization is sufficient. In such applications, range-free localization techniques are being used as low cost alternative to the range based localization techniques. All nodes in a sensor network cannot be made location aware, as it may escalate the cost of sensor network deployment. Therefore, to reduce the cost, very few location aware nodes, known as anchor nodes are deployed in the sensor network, all other nodes need to determine their location with the help of anchor nodes. Therefore, localization in wireless sensor network is to determine the geographical position of sensor nodes, based on the known position of anchor nodes. In this paper, range-free centroid schemes, based on fuzzy logic inference approach have been proposed. The proposed schemes have been compared with existing rangefree centroid based localization schemes through extensive simulations. A new cooperative localization technique based on fuzzy logic inference approach has also been proposed, which require deployment of very less number of anchor nodes in sensing field as compared to the conventional techniques; yet it provides required accuracy in localization of sensor nodes. The cooperative scheme requires very small number of location aware anchor nodes to be deployed, which reduces the cost of sensor network deployment. The simulation results demonstrate that through proposed cooperative localization technique, the desired accuracy in localization can be achieved even by use of few number of anchor nodes.
\end{abstract}

Keywords: Centroid localization, Edge weight, Fuzzy logic system, Range-free localization, Wireless Sensor Networks, Sugeno fuzzy logic inference, Mamdani fuzzy logic inference, ANFIS, Cooperative localization.

\section{Introduction}

Wireless sensor networks (WSNs) are emerging as a promising technology for ubiquitous sensing, monitoring and controlling home, office, city and environment for large number of applications [1]. Recent advances in wireless communications and electronics have enabled the development of low cost, low power and multifunctional sensors, which when networked wirelessly provide broad spectrum of applications in defense, habitant monitoring, target tracking, traffic monitoring, etc. [3,5]. Sensor networks can be deployed to monitors hostile

DOI : $10.5121 /$ ijcnc.2011.3607 
and hard locations. Localization (location estimation) capability is essential in most of the WSN applications, where, the sensed data is meaningless without the knowledge of precise location from where it is obtained. In addition, location awareness plays an important role in designing energy efficient routing protocols for wireless sensor networks [22, 23, 24]. Location of sensor nodes can be obtained either by placing the sensor nodes at points with known coordinates or by deployment of global positioning systems (GPS) on every sensor node. Since, the sensor nodes are randomly thrown in the sensing field in large numbers; they cannot be placed at the known location. Also, deployment of GPS on every sensor node is not feasible as it will escalate the cost of sensor network deployment. Therefore, wireless sensor localization techniques are used to estimate the location of sensor nodes in the network using the apriori location knowledge of few specific sensor nodes deployed in sensing field, known as anchor nodes. The anchor nodes can obtain their location using global positioning system (GPS), or by placement at points with known coordinates. In application requiring knowledge of global coordinate systems, the anchors determine the location of sensor nodes with reference to the global coordinate system and the application where a local coordinate system is sufficient, the position of sensor nodes are referred to the local coordinate system of network. Many localization algorithms exist in the literature for location estimation of sensor nodes in WSN [1, $6,12,18,23,24]$. The localization algorithms can be divided into two categories: (i) rangebased localization techniques [7, 10, 18], and (ii) range-free localization techniques [4, 9, 12]. Range-based localization is defined by protocols that use absolute point-to-point distance estimates (range) or angle estimates for calculating the location. These methods utilize the measurement such as angle of arrival (AOA), time of arrival (TOA), time difference of arrival (TDOA) and received signal strength (RSS) profiling. In contrast, range-free localization schemes make no assumption about the availability or validity of such information. Normally range-based schemes have higher location accuracy than the range-free localization schemes, but are hardware intensive [2] and escalate the cost of sensor nodes. Because of the hardware limitations of WSN devices, solutions in range-free localization are being pursued as a costeffective alternative to more expensive range-based approaches. Bulusu et al. proposed a simple and economic range-free localization technique, based on proximity based centroid algorithm [9]. In this method a sensor node localizes itself by calculating the centroid position of all the adjacent connecting anchor nodes. Though simple and economic, the localization estimates of this method are quite poor and have large error in measurement. To improve the performance of simple centroid algorithm, an enhanced weighted centroid algorithm was proposed by Kim and Kwon [6]. The method provides good accuracy in localization, but the accuracy of localization depends upon optimized selection of edge weights, which are selected heuristically in the proposed method. Y. Sukhyun et al. used Takagi Sugeno Kang (TSK fuzzy) modeling for calculating the weights of anchor nodes [15]. In this method, genetic algorithm is used to optimize the fuzzy membership function to obtain optimized edge weight based on the received signal strength (RSS). Y. Sukhyun et al. also proposed neural network approach for calculation of weights for centroid algorithm [14]. Both of these approaches provide acceptable accuracy in localization of sensor nodes, but the computational complexity is quite high for resource constrained sensor nodes.

This paper contributes to range- free localization in wireless sensor networks by proposing new weighted centroid based, range free localization techniques: (i) ANFIS trained Sugeno weighted centroid localization, (ii) Combined Sugeno-Mamdani fuzzy localization, and (iii) Centroid based range fee cooperative localization technique. The weights of anchor nodes have been 
obtained based on the Received Signal Strength Information (RSSI), which requires no additional hardware in sensor node, as it is available as part of physical layer of sensor node. In case of ANFIS trained Sugeno weighted centroid localization technique; fuzzy membership functions have been optimized using ANFIS trained Sugeno fuzzy inference system to calculate the weights of anchor nodes. Further, weighted centroid method has been used to obtain the localization information of sensor nodes. In case of Combined Sugeno-Mamdani fuzzy approach of localization, the edge weights of anchor nodes are calculated by averaging the edge weights obtained from Sugeno and Mamdani fuzzy approach. The accuracy of localization and extent of localization i.e. percentage of nodes localized in sensing field after completion of localization cycle depends upon number of location aware anchor nodes deployed in the sensing field. Reduction in number of anchor nodes lowers the accuracy of localization as well as extent of localization in the network. Since, each location aware anchor node is equipped with GPS system to ascertain its position; it escalates the cost of anchor node. Therefore, the overall cost of sensor network depends on the number of anchor nodes deployed in the sensing field. Cooperative localization scheme is proposed to considerably reduce the requirement of location aware anchor nodes, thus reducing the cost of sensor network deployment, without compromising the localization accuracy and extent of localization in WSN. Cooperative localization technique consists of cycles of localization, in each cycle, the nodes which are within radio range of three or more anchor nodes, localize themselves and once localized; they act as anchor node to localize remaining nodes during next cycle.

The rest of the paper is organized as follows In Section 2 centroid localization, weighted centroid localization and fuzzy logic system is introduced. In Section 3, proposed ANFIS trained Sugeno and Sugeno-Mamdani localization techniques are presented. Section 4 discusses about the simulation tool, simulation environment, and simulation results. The simulation results of proposed localization techniques are compared with existing centroid based localization techniques in this section. In Section 5 range-free cooperative localization scheme and its simulation are discussed and finally conclusions are drawn in Section 6.

\section{Background}

\subsection{Centroid and Weighted Centroid Localization}

Centroid localization technique is a range-free localization, which uses the knowledge of adjacent connected anchor node position $\left(\mathrm{X}_{\mathrm{i}}, \mathrm{Y}_{\mathrm{i}}\right)$ to estimate the location of sensor node [9]. In this method, the anchor nodes transmit a beacon containing their respective location information. The sensor node computes its position $\left(\mathrm{X}_{\mathrm{est}}, \mathrm{Y}_{\mathrm{est}}\right)$ as centroid of the positions of all the adjacent connected anchor nodes to it as:

$$
\left(X_{e s t}, Y_{e s t}\right)=\left(\frac{X_{1}+\ldots .+X_{N}}{N}, \frac{Y_{1}+\ldots .+Y_{N}}{N}\right)
$$

Where, $\left(\mathrm{X}_{\mathrm{est}}, \mathrm{Y}_{\mathrm{est}}\right)$ represents the estimated position of the sensor node and $\mathrm{N}$ is the number of adjacent connected anchor nodes to the sensor node. The method of location estimation using centroid method is quite simple and economic, but the location estimation results of this method are very poor and localization error is very high, which is unacceptable in many applications. An improved version of centroid method is weighted centroid localization [6]. In this method, location of sensor node is calculated by using edge weights of anchor nodes connected to the sensor node, and each sensor node computes its position $\left(\mathrm{X}_{\mathrm{est}}, \mathrm{Y}_{\mathrm{est}}\right)$ as: 
International Journal of Computer Networks \& Communications (IJCNC) Vol.3, No.6, November 2011

$$
\left(X_{e s,}, Y_{e s t}\right)=\left(\frac{w_{l} \cdot X_{l}+\ldots .+w_{n} \cdot X_{n}}{\sum_{i=1}^{n} w_{i}}, \frac{w_{l} \cdot Y_{l}+\ldots .+w_{n} \cdot Y_{n}}{\sum_{i=1}^{n} w_{i}}\right)
$$

Where, $w_{i}$ is the edge weight of $i^{\text {th }}$ anchor node connected to the sensor node. The edge weight is decided based upon the proximity of anchor node to the sensor node. Performance of this approach highly depends on the optimization of edge weights.

\subsection{Fuzzy Logic Inference}

Fuzzy logic inference is the process of formulating the mapping from a given input to an output, using fuzzy logic. The mapping then provides a basis, from which decisions can be made [19]. The process of fuzzy inference involves the membership functions, logical operations, and If-Then Rules. Fuzzy logic inference system can be used for designing of edge weights for anchor nodes $[14,15]$. It consists of a fuzzifier, some fuzzy If-Then rules, fuzzy inference engine, and defuzzifier. Fuzzy inference system can be implemented in two ways: Mamdani-type [20] and Sugeno-type [21]. Mamdani's fuzzy inference method is the most commonly seen fuzzy methodology. It expects the output membership functions to be fuzzy sets. After the aggregation process, there is a fuzzy set for each output variable that needs defuzzification. It's possible, and in many cases much more efficient, to use a single spike as the output membership function rather than a distributed fuzzy set, known as a singleton output membership function and it can be thought of as a pre-defuzzified fuzzy set. Sugeno or TakagiSugeno-Kang method of fuzzy inference is similar to the Mamdani method in many respects. The first two parts of the fuzzy inference process, fuzzifying the inputs and applying the fuzzy operator, are exactly the same. The main difference between Mamdani and Sugeno is that the Sugeno output membership functions are either linear or constant.

A fuzzy rule can be written as the following statement:

$$
\text { Rule i: } \quad \text { If } \mathrm{x}_{1} \text { is } W_{1}^{i} \text { and } \mathrm{x}_{2} \text { is } W_{2}^{i} \text { and ......... } \mathrm{x}_{\mathrm{n}} \text { is } W_{n}^{i} \text { Then } \mathrm{y} \text { is } y^{i}
$$

Where, Rule $i(i=1,2, \ldots ., N)$ denotes the $i^{\text {th }}$ implication and $N$ is the number of rules; $x_{j}(j=$ $1,2, \ldots, n)$ is input variables of the Fuzzy Logic System (FLS); $y_{i}$ is a singleton; $W_{j}^{i}$ are fuzzy membership functions, which represent the uncertainty in the reasoning. When we use the product inference, center-average and singleton fuzzifier, the output of the fuzzy system for an input $X=\left(\mathrm{x}_{1}, \mathrm{x}_{2} \ldots, \mathrm{x}_{\mathrm{n}}\right)$ can be expressed as [14]:

$$
y=\frac{\sum_{i=1}^{N} a_{i} y_{i}}{\sum_{i=1}^{N} a_{i}}
$$

Where, $a_{i}$ implies the overall truth value of the premise of the $\mathrm{i}^{\text {th }}$ implication, and are computed

$$
a_{i}=\prod_{j=l}^{n} W_{j}^{i}\left(x_{i}\right)
$$


International Journal of Computer Networks \& Communications (IJCNC) Vol.3, No.6, November 2011

\section{Fuzzy Logic Based Localization Technique}

Fuzzy logic can be used to decide the edge weights of sensor nodes for weighted centroid localization technique. This section describes the proposed localization methods (i) ANFIS trained Sugeno weighted centroid localization, and (ii) Combined Sugeno-Mamdani fuzzy localization. In ANFIS trained Sugeno method, the edge weights are obtained by Sugeno fuzzy inference method and are optimized by using ANFIS training to calculate the weight of anchor nodes. In case of Combined Sugeno-Mamdani fuzzy approach, the edge weights of anchor nodes are calculated by averaging the edge weights obtained from Sugeno and Mamdani fuzzy approach. Received signal strength information (RSSI) of adjacent connected anchor nodes is used to obtain the weights of anchor nodes in both cases. The weighted centroid method is used for localization of sensor nodes, using the edge weights obtained by proposed approaches.

\subsection{Network Model}

Wireless sensor network consists of a set of sensor nodes deployed randomly in large area to monitor the parameters of interest. These nodes are categorized as: anchor nodes and normal sensor nodes. Anchor nodes are special type of nodes embedded with GPS or other facility to obtain their position within the network. If feasible, these nodes can also be placed manually at known positions within the network. It is assumed that $\mathrm{n}$ numbers of anchor nodes are deployed in the sensing field. The position of anchor nodes is assumed as $\left(X_{1}, Y_{1}\right),\left(X_{2}, Y_{2}\right), \ldots,\left(X_{n}, Y_{n}\right)$. Anchor nodes transmit periodic beacon signals containing information regarding their respective positions with overlapped region of coverage. Sensor nodes are deployed in the sensing field, with randomly distributed positions. These sensor nodes localize themselves with the help of beacon signals, transmitted by the anchor nodes. Each sensor node collects the received signal strength information (RSSI) of all connected adjacent anchor nodes through beacon signal and RSSI is used to obtain the edge weights of the anchor nodes for weighted centroid localization. Time division multiplexing (TDM) technique is used to avoid interference of beacons transmitted by neighboring anchor nodes. The radio transmission range of all nodes is assumed to be identical and perfectly spherical.

\subsection{Calculating the Edge Weights using Fuzzy Inference System}

The sensor node collects the received signal strength information (RSSI) values of all connected adjacent anchor nodes. The edge weights of anchor nodes need to be calculated for finding sensor node position. In this paper, fuzzy systems with symmetrical trapezoidal membership function for input (RSSI) and output (weight) have been used. The input variable is the RSSI from anchor node and can take values in the interval [0, RSSI ${ }_{\max }$ ], where, $\mathrm{RSSI}_{\max }$ is the maximum RSSI value. The output variable is the edge weight of each anchor node for a given sensor node and can take value in the interval $\left[0, \mathrm{~W}_{\max }\right]$, where $\mathrm{W}_{\max }$ is the maximum weight. For modeling the fuzzy logic inference system (FLI), If-Then rules have to be considered, which follow the basic principle that if a sensor node senses high powered signal from an anchor node, the anchor node is likely to be in close proximity to the given sensor node and hence is assigned high weight. Conversely, if a sensor node is connected to an anchor node but senses low powered signal, the anchor node is likely to be far from the given sensor node and hence is assigned a low weight. Consequently, the fuzzy rule bases are used and tuned the membership functions $[14,15]$ as shown in Figure 1 
International Journal of Computer Networks \& Communications (IJCNC) Vol.3, No.6, November 2011

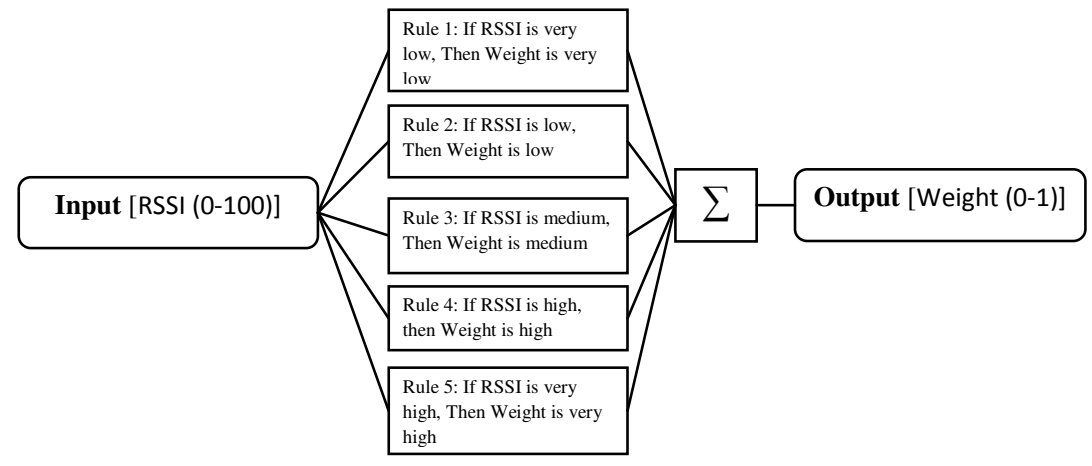

Figure 1. Weight calculation for input RSSI

\subsubsection{Edge weight calculation using Mamdani fuzzy inference system}

For Mamdani FLI based localization method, fuzzy logic system has been modeled using Mamdani fuzzy inference system. Where, the input (RSSI) and output (Weights) space have been decomposed into five symmetrical trapezoidal membership functions namely: very low, low, medium, high, and very high, as shown in Figure 2.

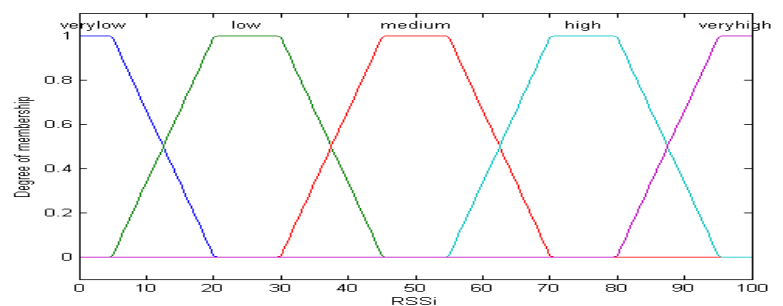

(a)

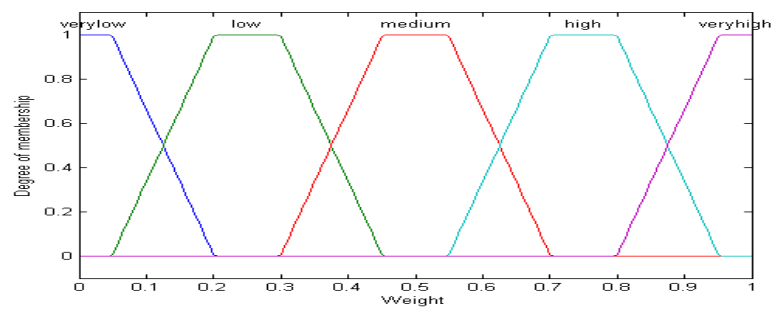

(b)

Figure 2. Mamdani fuzzy membership function of (a) RSSI, and (b) weight

\subsubsection{Edge weight calculation using Sugeno fuzzy inference system}

For Sugeno FLI based localization method, fuzzy system has been modeled using Sugeno method of fuzzy inference [8]; which is similar to the Mamdani method in many aspects. The first two parts of the fuzzy inference process, fuzzifying the inputs and applying the fuzzy operator, are exactly the same. 


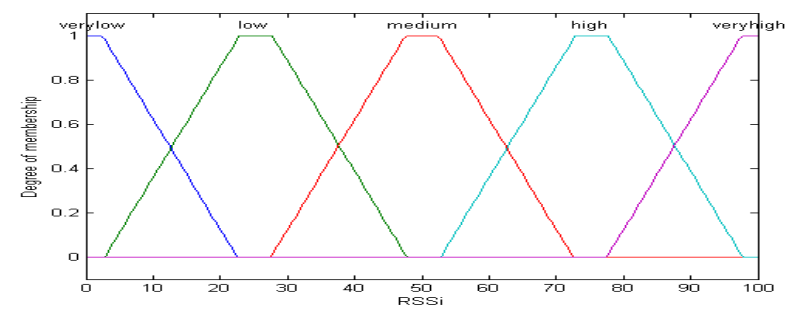

Figure 3. Sugeno fuzzy membership function of RSSI

The main difference between Mamdani and Sugeno is that the Sugeno output membership functions are either linear or constant. In our approach we have considered liner output membership function and have decomposed the input (RSSI) into five symmetrical trapezoidal membership functions namely: very low, low, medium, high, and very high, as shown in Figure 3. The output has been decomposed into five linear symmetrical functions namely: very low, low, medium, high, and very high. Sugeno systems do not have the output membership function plot. The defuzzification is considered to be weighted average.

\subsection{Proposed ANFIS Trained Sugeno Fuzzy System}

The acronym ANFIS derives its name from Adaptive Neuro-Fuzzy Inference system [8]. Using a given input/output data set, ANFIS can constructs a fuzzy inference system (FIS) whose membership function parameters are tuned (adjusted) using either a back propagation algorithm alone or in combination with a least squares type method. This adjustment allows our fuzzy systems to learn from the data we are modeling [16]. In our approach, the Sugeno fuzzy inference system is trained using the ANFIS, which model the system to get the linear edge weight related to the RSS information as shown in Figure 4.

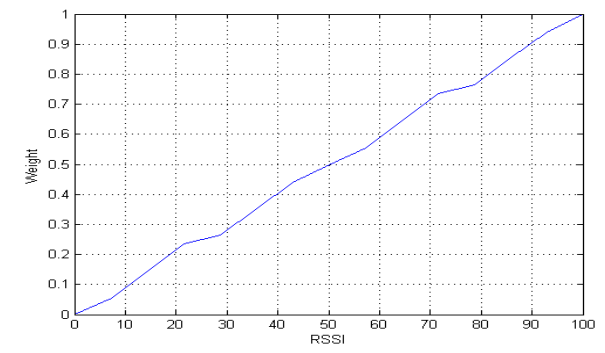

(a)

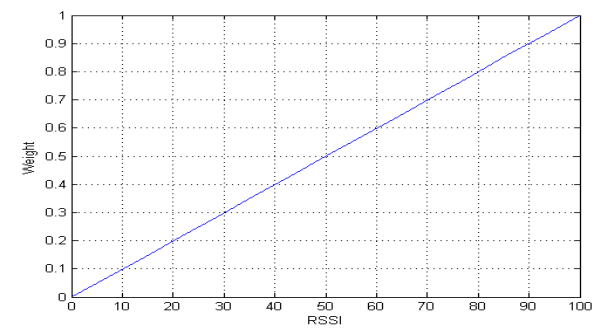

(b)

Figure 4. Relationship between RSSI and (a) weight for Sugeno FLI, and (b) weight for ANFIS trained Sugeno

\subsection{Proposed Combined Sugeno-Mamdani Localization Approach}

A combined Sugeno-Mamdani localization approach has been proposed for calculating the sensor node position. If the estimated node coordinates for Sugeno and Mamdani system are 
$\left(\mathrm{X}_{\text {st-sug }}, \mathrm{Y}_{\text {est-sug }}\right)$ and $\left(\mathrm{X}_{\text {est-mam }}, \mathrm{Y}_{\text {est-mam }}\right)$ respectively, then the estimated position of sensor node can be calculated by taking the average of coordinates as per the following formula [21]:

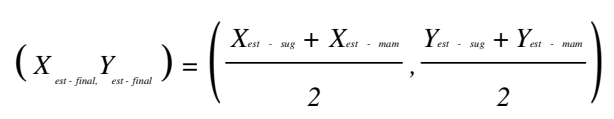

\subsection{Performance Evaluation}

For evaluating the proposed schemes and comparing with existing methods, following two performance indices have been used:

\subsubsection{Instantaneous localization error}

Instantaneous location error is defined as the distance between the instantaneous estimated position and the actual position of sensor node. It gives the actual localization error of sensor node in the sensing field. Instantaneous localization error is given as:

$$
\text { Localization Error }=\sqrt{\left(\mathrm{X}_{\text {est }}-\mathrm{X}_{\mathrm{a}}\right)^{2}+\left(\mathrm{Y}_{\text {est }}-\mathrm{Y}_{\mathrm{a}}\right)^{2}}
$$

Where, $\left(\mathrm{X}_{\text {est }}, \mathrm{Y}_{\text {est }}\right)$ is the estimated position of sensor node while $\left(\mathrm{X}_{\mathrm{a}}, \mathrm{Y}_{\mathrm{a}}\right)$ is the actual position of sensor node.

\subsubsection{Average location error}

Average location error is used as parameter for performance evaluation. It is defined as the average distance between the estimated position and the actual position of all sensor nodes,

$$
\text { Average Localization Error }=\frac{\sum \sqrt{\left(\mathrm{X}_{\text {est }}-\mathrm{X}_{\mathrm{a}}\right)^{2}+\left(\mathrm{Y}_{\text {est }}-\mathrm{Y}_{\mathrm{a}}\right)^{2}}}{\text { Number of sensor nodes }}
$$

\section{Range-free Cooperative Localization}

Cooperative localization requires very few anchor nodes sensor node for localization in the sensor network. The flow chart for cooperative localization is given in Figure 5. During first cycle all anchor node broadcast their position. All non-anchor nodes which are in range of three or more anchor nodes; localize themselves using either simple centroid or proposed combined Sugeno-Mamdani localization approach. Those nodes, which are not in range of three or more anchor nodes, do not participate in localization process during initial cycles of localization, as accuracy of localization in centroid method depends upon the number of adjacent anchor node, used for localization of a node. The location accuracy in cooperative localization is highly dependent on the location accuracy of nodes during initial cycles. As the nodes localized in particular cycle acts as anchor nodes for all subsequent cycles. Therefore, all those nodes which are inaccurately localized during initial cycles, when used as anchor nodes, spread the localization error thorough out the network. Non-anchor nodes which are localized in first cycle become anchor node and broadcast their location information beacon for next localization cycle. Again, those non-anchor nodes which are in the range of more than three anchor nodes localize themselves. The process is repeated again and again. If, in a particular cycle none of the nodes are in range of three or more than three anchor nodes, then the nodes are allowed to be localized with two nodes. The process is continued till all the anchor nodes get localized. 


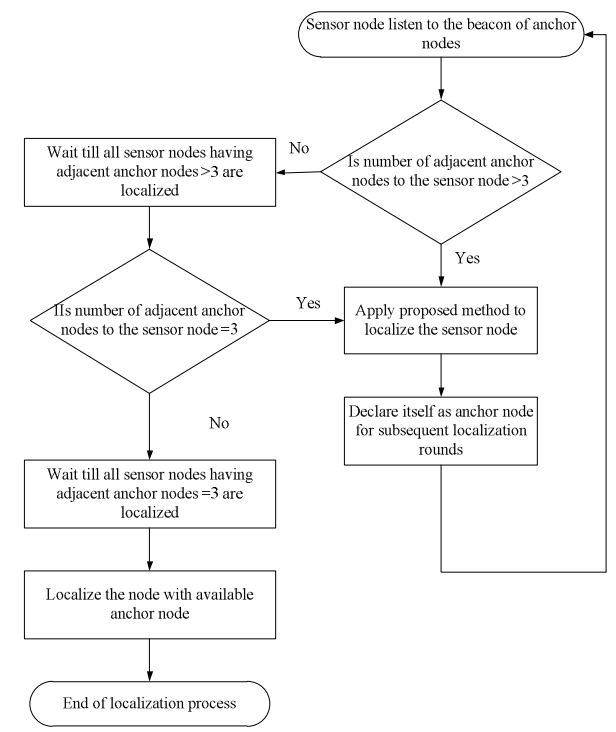

Figure 5. Flow Chart for Location Estimation in cooperative localization

\section{Simulation Setup and Results}

MATLAB has been used for performance evaluation of the proposed schemes. The simulation has been carried out number of times and average results of simulations are used for performance evaluation. Parameters used for simulation are cited in Table 1.

Table 1. Simulation parameters

\begin{tabular}{|l|c|}
\hline Parameter & Value \\
\hline Number of sensor nodes & 60 \\
\hline Number of anchor nodes (for non-cooperative localization) & 121 \\
\hline Number of anchor nodes (for cooperative localization) & 25 \\
\hline Area of sensing field & $10 \times 10 \mathrm{~m}^{2}$ \\
\hline Transmission range of nodes & $8.94 \mathrm{~m}$ \\
\hline
\end{tabular}

The Friis free space radio model is used to determine the received signal strength value at the sensor node [14]. The received signal strength at the sensor node is given by:

$$
R_{i j}=\left(k d_{i j}^{-a}\right)
$$

Where $R_{i j}$ is the received signal strength value received by $i^{\text {th }}$ sensor node, transmitted by $j^{\text {th }}$ adjacent anchor node, $\mathrm{k}$ is a constant which takes into account carrier frequency and transmitted power, $d_{i j}$ is the distance between the $\mathrm{i}^{\text {th }}$ sensor node and the $\mathrm{j}^{\text {th }}$ adjacent anchor node and $\alpha$ is the attenuation exponent. Here, we have used $\mathrm{k}=50$ and $\alpha=2$ (Direct path radio model).

In this paper, three existing localization techniques have been used for comparison against the proposed schemes using the performance metrics explained in Section 3.5. Figures from 6 to 10 show the result of location estimation and localization error of these methods respectively. 
International Journal of Computer Networks \& Communications (IJCNC) Vol.3, No.6, November 2011
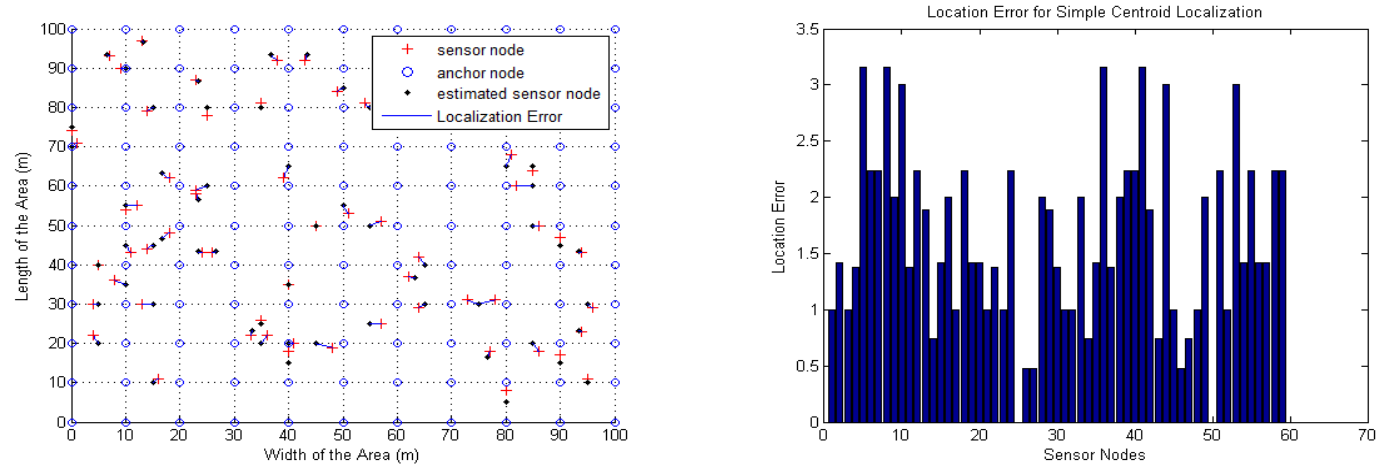

Figure 6. Simulation results of location error in case of Simple Centroid Scheme
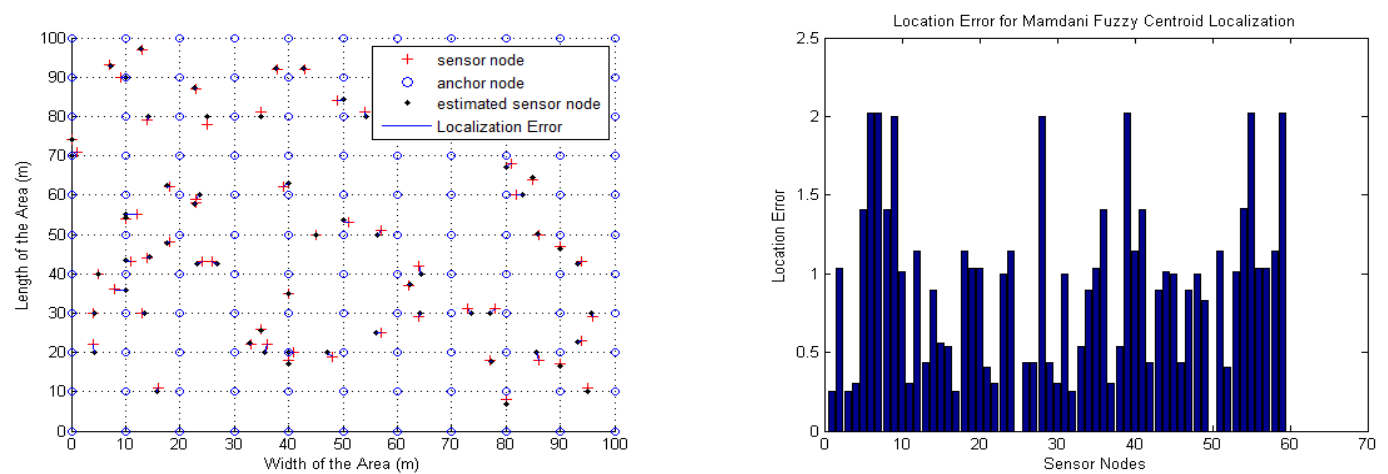

Figure 7. Simulation results of location error in case of Mamdani FLI scheme
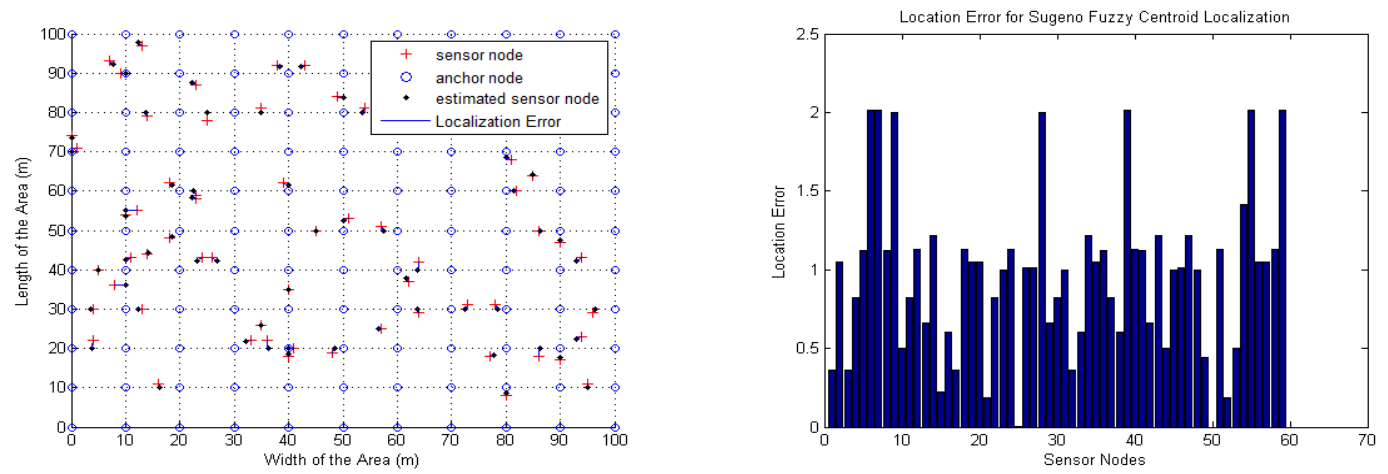

Figure 8. Simulation results of location error in case of Sugeno FLI scheme 
International Journal of Computer Networks \& Communications (IJCNC) Vol.3, No.6, November 2011
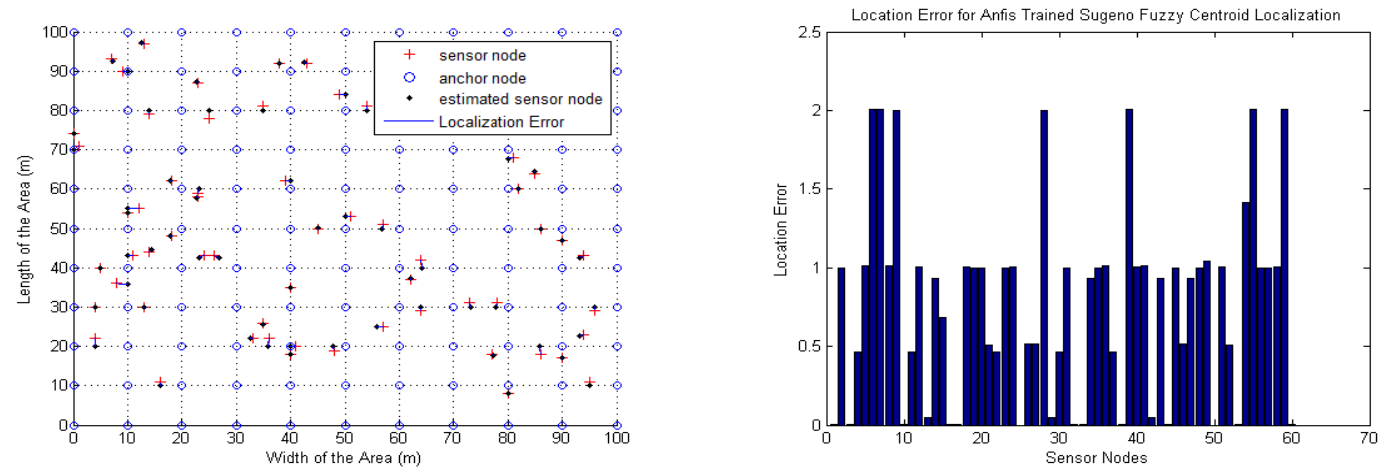

Figure 9. Simulation results of location error in case of ANFIS trained Sugeno scheme
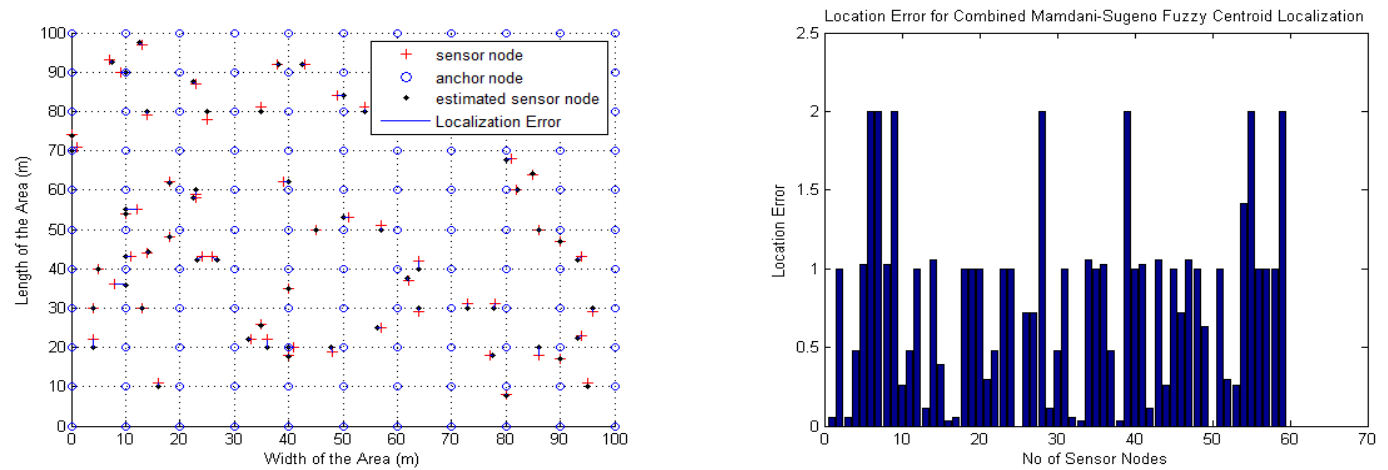

Figure 10. Simulation results of location error in case of combined Sugeno-Mamdani scheme

The simulation results are summarized in Table 2.

Table 2. Comparison results for different approaches

\begin{tabular}{|l|c|c|c|}
\hline \multicolumn{1}{|c|}{ Approaches } & $\begin{array}{c}\text { Max. error } \\
\text { (meters) }\end{array}$ & $\begin{array}{c}\text { \% of anchor } \\
\text { nodes }\end{array}$ & $\begin{array}{c}\text { Average error } \\
\text { (meters) }\end{array}$ \\
\hline Simple centroid & 3.1623 & 66.8 & 1.6080 \\
\hline Mamdani fuzzy & 2.0211 & 66.8 & 0.8956 \\
\hline Sugeno fuzzy & 2.0108 & 66.8 & 0.9462 \\
\hline Proposed Combined Sugeno-Mamdani & 2.0050 & 66.8 & 0.7664 \\
\hline
\end{tabular}

It can be seen from the simulation results that proposed ANFIS trained Sugeno and Combined Sugeno-Mamdani approaches provide highly accurate localization results in comparison to conventional schemes. However, all centroid formula based range-free localization approaches require high percentage of anchor node deployment. The accuracy of location depends on the number of anchor node deployment. Figure 11 shows the variation in localization error with variation in number of anchor nodes. For this simulation 60 sensor nodes (non-anchor) distributed randomly and respectively 60 to 240 anchor nodes are distributed randomly in a sensing field of $10 \times 10 \mathrm{~m}^{2}$. 


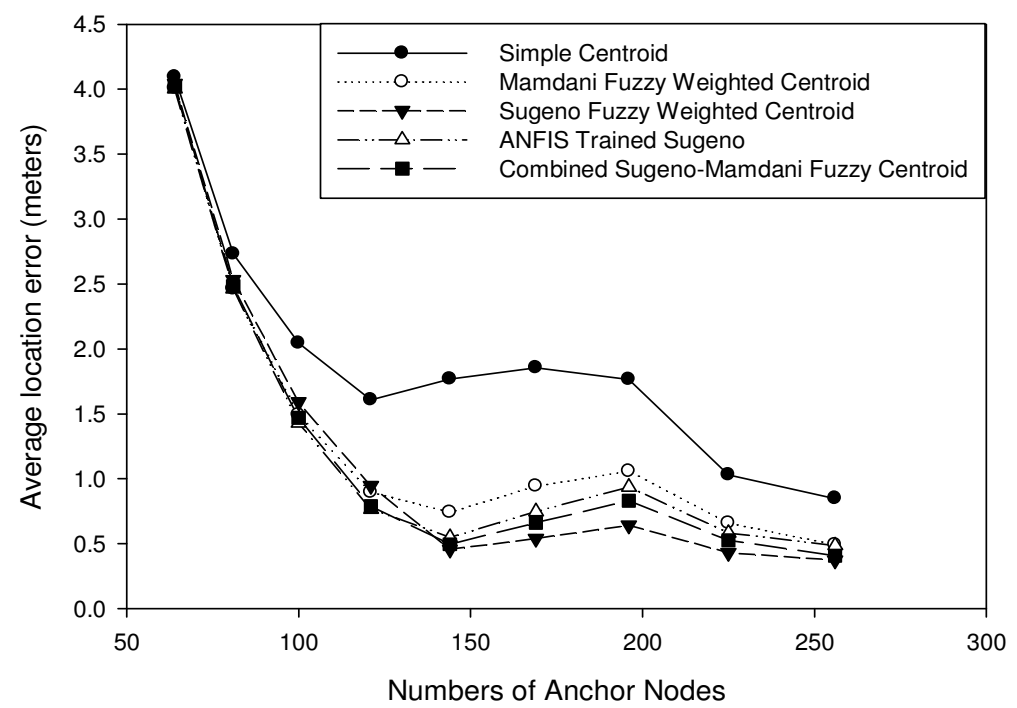

Figure 11. Simulation results of average localization error vs. number of anchor nodes

Figure 12 and 13 show the localization error for cooperative localization. Table 4 summarizes the comparison results of these approaches. It can be seen from the simulation results that localization error in the range-free cooperative localization is higher than the simple centroid approach. But the cooperative approach can provide acceptable results even by using less number of anchor nodes in the sensing field.
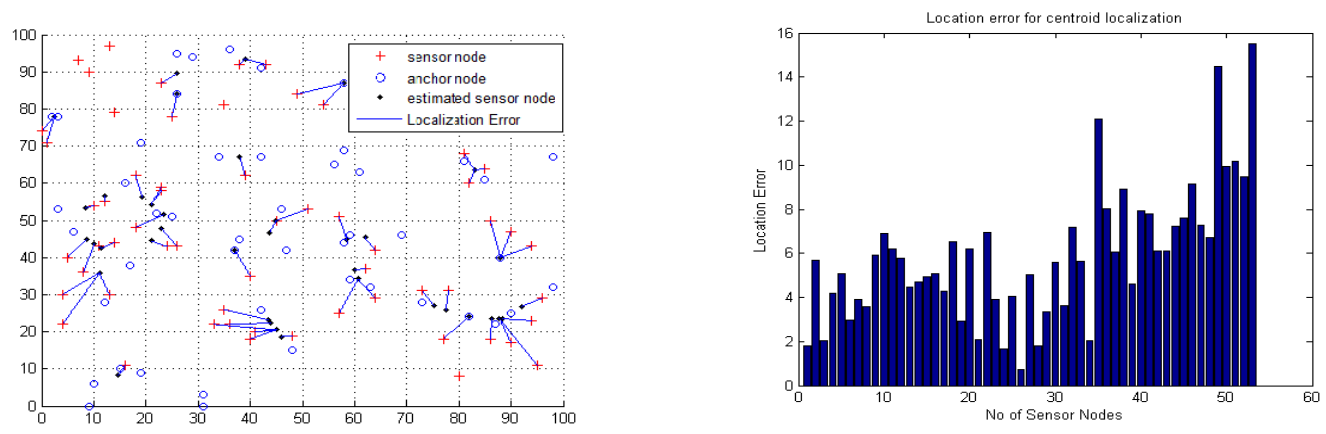

Figure12. Simulation results of location error in case of simple centroid scheme
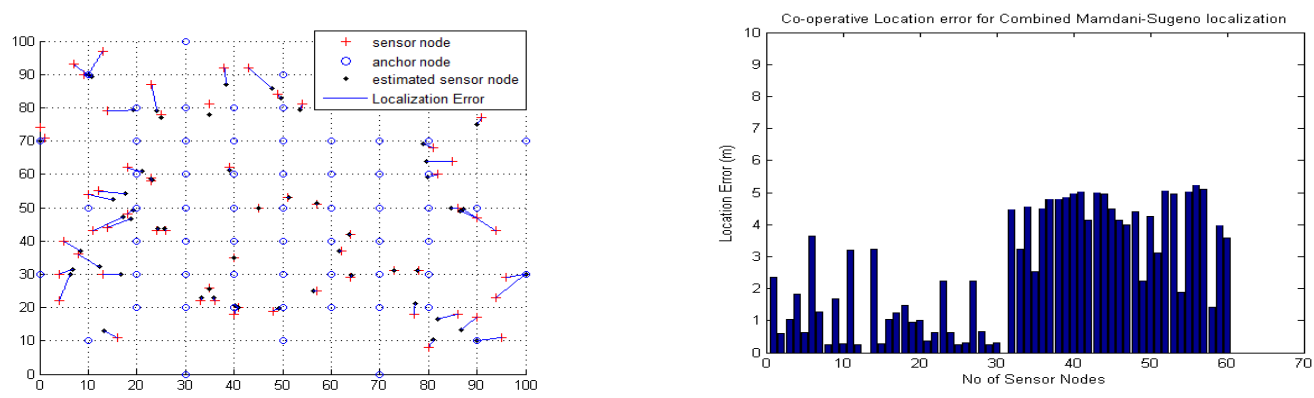

Figure 13. Simulation results of location error in case of combined Sugeno-Mamdani scheme 
The simulation results show that localization accuracy is substantially improved when cooperative localization is carried out with proposed combined Sugeno-Mamdani approach. It can be seen that the number of anchor nodes used in case of cooperative localization is much less than those used in simple range-free localization approaches. This reduces the cost of sensor network deployment.

Table 4. Comparison results for cooperative localization

\begin{tabular}{|c|c|c|c|}
\hline Approaches & $\begin{array}{c}\text { Max. error } \\
\text { (meters) }\end{array}$ & $\begin{array}{c}\text { \% of anchor } \\
\text { nodes }\end{array}$ & $\begin{array}{c}\text { Average error } \\
\text { (meters) }\end{array}$ \\
\hline Simple centroid & 9.84 & 23.8 & 3.98 \\
\hline $\begin{array}{c}\text { Proposed Combined Cooperative Sugeno- } \\
\text { Mamdani }\end{array}$ & 5.4 & 23.8 & 1.74 \\
\hline
\end{tabular}

\section{Conclusion}

Given the inherent constrains of the sensor devices and estimation accuracy desired by location dependent application, range-free localization are regarded as cost effective solution for sensor node localization. The RSS information between sensor nodes and its neighbor anchor nodes can be used to estimate the positions without any complicated hardware. Fuzzy logic system is the main component of our proposed schemes. In case of ANFIS trained Sugeno weighted centroid localization technique; fuzzy membership functions have been optimized using ANFIS trained Sugeno fuzzy inference system to calculate the weight of anchor nodes. In SugenoMamdani approach, which is less computation intensive, a combined Mamdani and Sugeno fuzzy system is used to determine the edge weight of anchor node and to enhance the location estimation accuracy of sensor nodes in the sensor network. The proposed approaches are simulated using MATLAB. The results are compared with existing techniques through extensive simulations. The simulation results demonstrate the effectiveness of the proposed schemes in comparison to the previous methods. However, these approaches require deployment of large number of location aware anchor nodes. The accuracy of localization is highly dependent upon the number of anchor nodes deployed in the network. Since, the anchor nodes require GPS equipment for localization; it costs more than the ordinary sensor nodes. Therefore, cooperative localization scheme is proposed, which provides required accuracy in localization with less number of anchor nodes, thus reducing the cost of sensor network deployment. The simulation results prove that the cooperative localization can be used for range-free localization scheme with required accuracy and minimum number of anchor node deployment.

\section{References}

[1] A. Pal, "Localization Algorithms in Wireless Sensor Networks: Current Approaches and Future Challenges," Network Protocols and Algorithms, Vol. 2, No.1, March 2010, pp. 45-73.

[2] A. Savvides, C. Han and M. B. Strivastava, "Dynamic Fine-Grained Localization in Ad-hoc Networks of Sensors," Proceedings of the $7^{\text {th }}$ Annual International Conference on Mobile Computing and Networking (MobiCom 01), July 2001, pp. 166 - 179

[3] G.Lukachan and M.A. Labrodor, "SELAR: Scalable Energy-Efficient Location Aided Rouing Protocol for Wireless Sensor Networks," Proceedings of LCN'04, Tampa, FL, Nov. 16-18, 2004. 
International Journal of Computer Networks \& Communications (IJCNC) Vol.3, No.6, November 2011

[4] C. Chong and S. P. Kumar, "Sensor Networks: Evolution, Opportunities, and Challenges," Proceedings of the IEEE, Vol. 91, No. 8, August 2003, pp. 1247 - 1256.

[5] D. Niculescu and B. Nath, "DV Based Positioning in Ad hoc Networks," Journal of Telecommunication Systems," Vol. 22, No. 1, January 2003, pp. 267-280.

[6] I.F. Akyildiz, W. Su, Y. Sankarasubramaniam, and E. Cayirci, "Wireless Sensor Networks: A Survey," Computer Networks, Vol. 38, No. 4, 2002, pp. 393-422.

[7] Kim S. Y. and Kwon O. H., "Location Estimation Based on Edge Weights in Wireless Sensor Networks," Journal of Korea Information and Communication Society, Vol. 30, No. 10A, 2005.

[8] L. Girod and D. Estrin, "Robust Range Estimation Using Acoustic and Multimodal Sensing," Proceedings of IROS 2001,Vol. 3,October 2001, pp. 1312 - 1320.

[9] M. Sugeno, "An Introductory Survey of Fuzzy Control," Information Science, Vol. 36, No. 1, July 1985, pp. 59-83.

[10] N. Bulusu, J. Heidemann, and D. Estrin, "GPS-less Low Cost Outdoor Localization for Very Small Devices," IEEE Personal Communication Magazine, Vol. 7, No. 5, October 2000, pp. 28-34.

[11] P. Bahl and V. N. Padmanabhan, "RADAR: An In-Building RF-Based User Location and Tracking System," Proceedings of the IEEE Infocom 2000, Vol. 2, March 2000, pp. 775 - 784.

[12] S. Meguerdichian, F. Koushanfar, M. Potkonjak, and M.B. Srivastava, "Coverage Problems in Wireless Ad-hoc Sensor Networks," Proceedings of IEEE Infocom 2001, Vol. 3, April 200, pp. 1380 $-1387$.

[13] T. He, C. Huang, B.M. Blum, J. A. Stankovic, and T. Abdelzaher, "Range-Free Localization Schemes for Large Scale Sensor Networks," Proceedings of the $9^{\text {th }}$ Annual International Conference on Mobile Computing and Networking (MobiCom 2003), September 2003, pp. 81-95.

[14] Y. B. Ko and N. H. Vaidya, "Location-Aided Routing (LAR) Mobile Ad Hoc Networks," Proceedings of The $4^{\text {th }}$ Annual International Conference on Mobile Computing and Networking (MobiCom 98), October 1998, pp. 66-75.

[15] Y. Sukhyun, L. Jaehun, and Wooyong, "A Soft Computing Approach to Localization in Wireless Sensor Networks," Expert Systems with Applications, Vol. 36, No. 4, May 2009, pp. 7552-7561.

[16] Yun S., Lee J., Chung W., and Kim E., "Centroid Localization Method in Wireless Sensor Networks Using TSK Fuzzy Modeling," International Symposium on Advanced Intelligent Systems," September 2005, pp. 971-974.

[17] Jang J.S.R., “ANFIS: Adaptive-Network-Based Fuzzy Inference System,” IEEE Transactions on Systems, Man and Cybernetics, Vol. 23, No. 3, 1993, pp. 665 - 685.

[18] T-F SHIH, W-T CHANG, "Positioning Strategy for Wireless Sensor Networks," Proceedings of $6^{\text {th }}$ WSEAS International Conference on Applied Electromagnetic, Wireless and Optical Communications (ELECTROSCIENCE '08), Trondheim, Norway, July 2-4, 2008, pp. 83-87.

[19] J. Arias, J. Lázaro, J. Jiménez, A. Zuloaga, and A. Astarloa, "Simulation Environment for Distance Based Location Algorithms in Wireless Sensor Networks," Proceedings of the $4^{\text {th }}$ WSEAS International Conference on Electronics, Hardware, Wireless and Optical Communications (EHAC'05), Salzburg, Austria, February 13-15 2005, pp. 492-248.

[20] P. Wolf, "Fuzzy Logic in Decision Making Process," Proceedings of $3^{\text {rd }}$ WSEAS/IASME International Conference on Dynamical Systems and Control (CONTROL '07), Arcachon, France, October 13-15, 2007, pp. 282-287.

[21] I. Ianchu and C. I. Popirlan, "Mamdani Fuzzy Logic Controller with Mobile Agents for Matching," Proceedings of Recent Advances in Neural Networks, Fuzzy Systems \& Evolutionary Computing (NNECFS'10), Iasi, Romania, June 13-15, 2010, pp. 117- 112. 
International Journal of Computer Networks \& Communications (IJCNC) Vol.3, No.6, November 2011

[22] Ashok Kumar, Vinay Kumar, and Vinod Kapoor, "Range Free Localization Schemes for Wireless Sensor Networks," Proceedings of $10^{\text {th }}$ WSEAS International Conference on Software Engineering, Parallel and Distributed Systems (SEPADS '11), Cambridge, UK, February 20-22, 2011, pp. 101106.

[23] Sanat Sarangi and Subrat Kar, "Performance Analysis Of An Improved Graded Precision Localization Algorithm For Wireless Sensor Networks," International Journal of Computer Networks \& Communications, Vol. 2, No. 4, pp. 150-159, July 2010.

[24] Ahmed M. Khedr and Hager Ramadan, "Effective Sensor Relocation Technique in Mobile Sensor Networks," International Journal of Computer Networks \& Communications, Vol. 3, No. 1, pp. 204-217, 2011. 